\title{
Analysis and interpretation of geodetic landslide monitoring data based on fuzzy systems
}

\author{
M. Haberler-Weber \\ Institute for Geodesy and Geophysics, Vienna University of Technology, Austria \\ Received: 18 July 2005 - Revised: 20 September 2005 - Accepted: 20 September 2005 - Published: 6 October 2005 \\ Part of Special Issue "Documentation and monitoring of landslides and debris flows for mathematical modelling and design of \\ mitigation measures"
}

\begin{abstract}
To place high precision geotechnical sensors exactly at the boundaries between blocks with different directions and rates of movement in a sliding area, it is important to detect these boundaries in a preceding step. An automated algorithm for the block detection based on fuzzy systems is presented. Combining objective geodetic indicators with fuzzy systems gives a powerful tool for the assessment of geodetic landslide monitoring data. The example of a landsliding area shows the applicability of the algorithm.
\end{abstract}

\section{Introduction}

Deformation measurements on landslides are a very important task in nowadays engineering geodesy. The classical idea is that the landslide has to be represented by discrete measuring points in such a way that the deformations of the sliding area can be reconstructed by the measured displacements of those points (Fig. 1, Pelzer, 1985). The measurements (tacheometric, GPS, ...) are done in at least two epochs, where the time interval between the epochs is dependent on the deformation rate.

In case some of the observed points are located in the stable area (serving as reference points), absolute displacements can be determined; otherwise only relative movements can be calculated.

In the classical geodetic deformation analysis, the geodetic measurements of at least two epochs are used to assess the displacements of the object points with statistical methods. So it can be stated, which points have statistically significant displacements. Most of the time, these displacement vectors and their graphical representation are the final results of the geodetic investigations, see Fig. 2 for an example (Pelzer, 1985).

Correspondence to: M. Haberler-Weber

(michaela.haberler-weber@tuwien.ac.at)
But for many applications, a deeper insight is necessary. One task is to find various blocks with similar pattern of movement based on a set of displacement vectors of discrete points given by the deformation analysis. This has been done up to now with different strategies, like further statistical analysis (Welsch, 1983) or pattern recognition techniques (Lehmkühler, 1993). But up to now it was not possible to automate this process.

Motivation for this work is the project OASYS, a multidisciplinary project for an integrated Optimization of landslide Alert SYStems. Landslide risk analysis and in the following a landslide alert system are of interest for scientists of many disciplines today. Therefore, the aim of OASYS is to collect as much information as possible from different disciplines (e.g. geodesy, geology, hydrology, remote sensing, ...), so that the information can be used within a knowledge based system to assess the further development of the sliding area and to define the landslide risk. At the end a clear decision should be possible to inform the affected inhabitants whether an evacuation is necessary.

The concept of OASYS is based on three different steps. In a first step large scale monitoring (e.g. by satellite techniques) has to be performed to find the landslide prone areas. Geodetic deformation measurements are carried out to get information on the block movement and on the boundaries between stable and unstable areas (see Fig. 3).

In a second step, in the areas found, high precision geotechnical measurement systems are installed. This multisensor system is logging data continously and can therefore feed a real-time alert system. The third step consists of the risk assessment and handling of the alert system. This final analysis for the risk assessment is supported by knowledge based systems, using multidisciplinary data from the preceding steps (see Fig. 4).

One task within this project is that - within step one and two - out of classical geodetic monitoring measurements, the boundaries between the stable and the unstable or between unstable areas moving with different velocities in different 


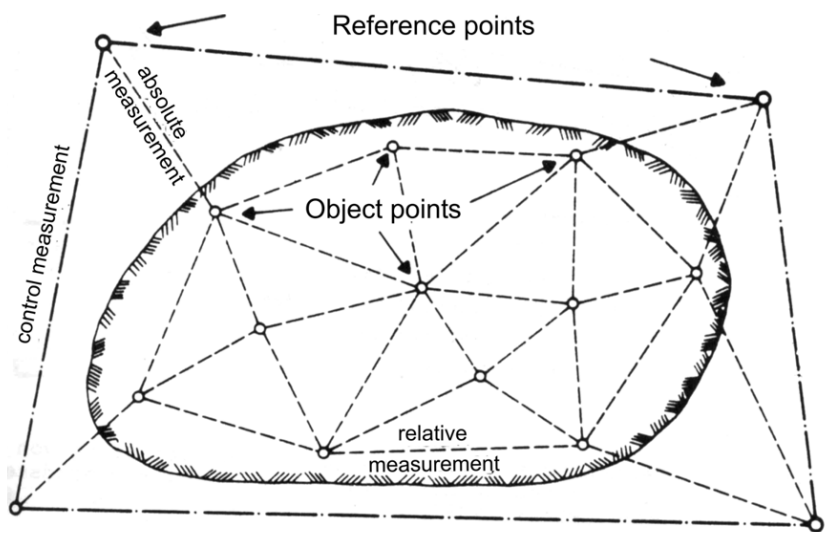

Fig. 1. Object points representing the object under investigation are connected by geodetic measurements, taken from Pelzer, 1985.

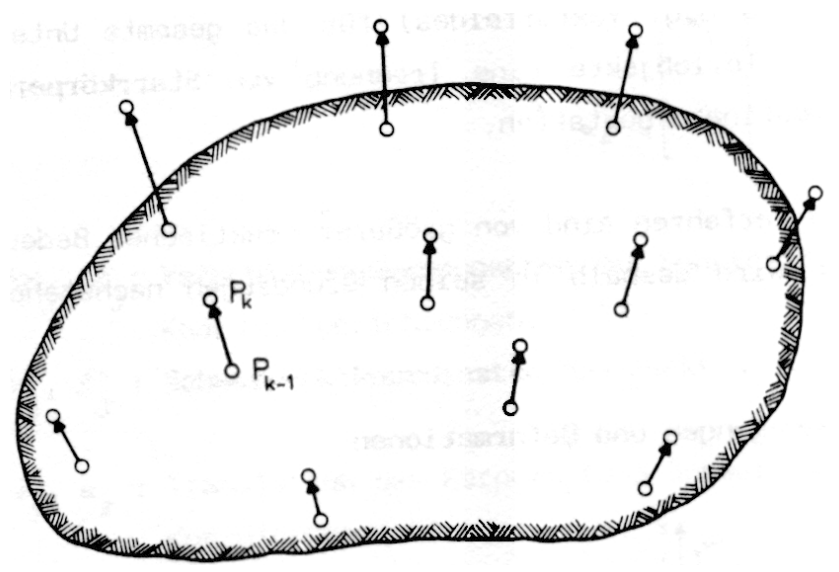

Fig. 2. Graphical representation of the displacement vectors for each object point as the result of the geodetic deformation analysis, taken from Pelzer, 1985.

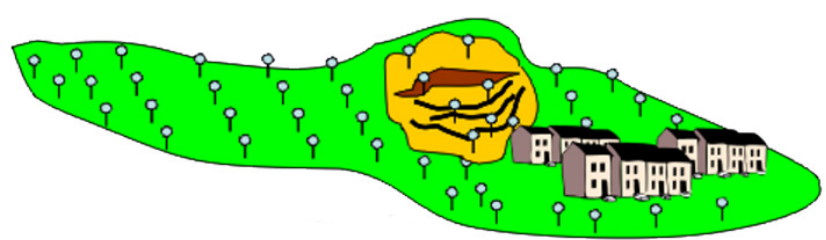

Fig. 3. Geodetic measurements connecting the object points shown here are used to find the critical areas of the landslide prone area.

directions have to be found, so that in the following step the geotechnical sensors mentioned above can be installed across these boundaries to get high precision monitoring data of the critical areas as an input for the knowledge based system for the risk assessment.

In the last few years, modern techniques like fuzzy systems, neural networks and knowledge based systems started to be used also in geodesy (see e.g. Heine, 1999; Miima, 2002; Wieser, 2002). One advantage of these methods is that they can reproduce the human way of thinking, so that problem solving is done in a rather intuitive way. Some un-

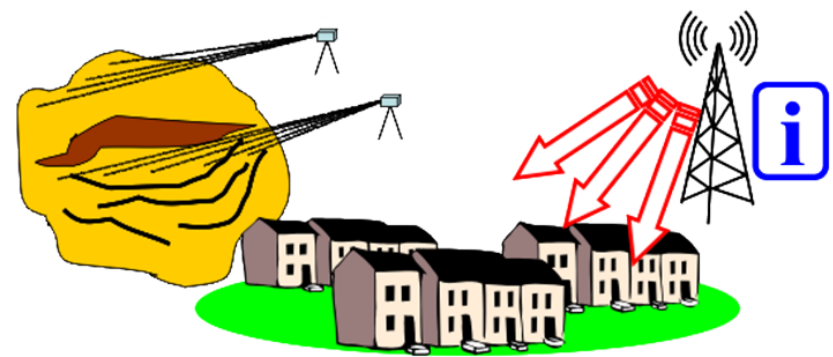

Fig. 4. Geotechnical monitoring is serving as the basis for the realtime alert system.

solved problems in geodesy are now further investigated, using these new techniques. Here a modern method for the automated detection of consistent block deformation based on fuzzy techniques is presented.

\section{Fuzzy systems}

With a fuzzy system the decision process of a human expert can be modelled. By defining input and output variables and rules connecting them the human way of thinking can be reproduced. Mathematical basics of the fuzzy theory cannot be given here, for further information see e.g. Kruse et al., 1995.

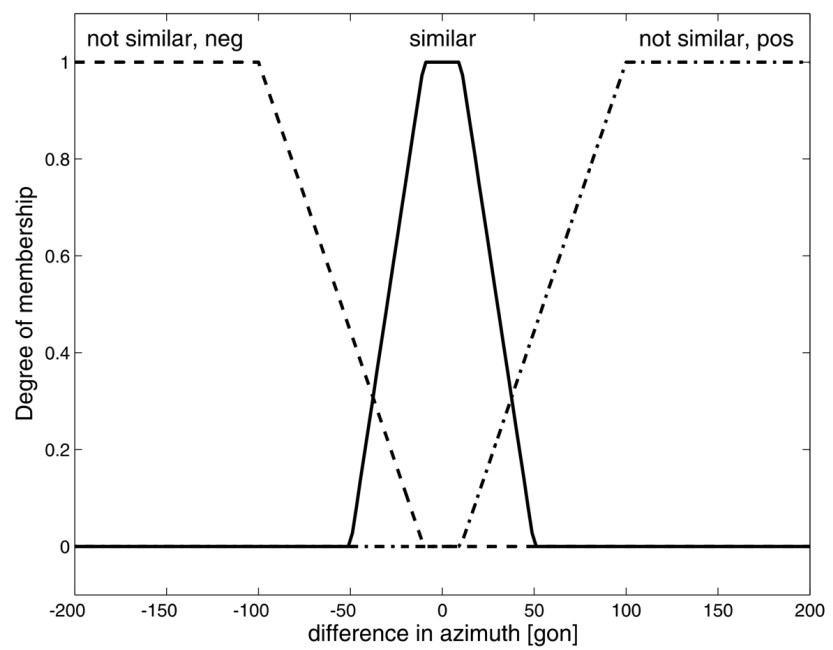

Fig. 5. Modelling of the input variable "similarity of direction" in the fuzzy systems by 3 linguistic terms.

The system presented here was implemented in Matlab ${ }^{\circledR}$. The first important step in implementing a fuzzy system is to find the suitable input variables and their membership functions. As an example, the input variable "similarity of direction" is modelled by the difference in azimuth of at least two vectors, represented by three linguistic terms: not similar (negative), similar, not similar (positive), covering the range of $(-200 ; 200)$ gon, see Fig. 5 (a more detailed description is given in Sect. 3.2). 
The next step is to implement the rules connecting the input variables and the output, defining the decision process. As an example it is shown by which rules the parameter "direction" is processed in the inference system.

If direction is

similar then block quality is very high

If direction is not

similar (negative) then block quality is very low

If direction is not

similar (positive) then block quality is very low

The number of rules in each fuzzy system depends of course on the number and the modelling of the input and output parameters. Each rule results in a fuzzy set; within the step of aggregation all these sets are combined (e.g. by the method of the maximum-operator), then the defuzzification is done with the help of e.g. the center of gravity-method to get one sharp output value as a result.

\section{Block detection algorithm}

At the end of the classical deformation analysis, a set of displacement vectors for the observed points on the landslide area is given. The task is to find groups of points with a similar pattern of movement so that the boundaries between these blocks can be identified.

Two different types of parameters are used here to do the block separation. First, geodetic influence factors are determined (cf. Sect. 3.1), second, the human way of assessing a graph of displacement vectors is copied by finding displacement vectors with similar direction and length, which are called "visual" influence factors here (cf. Sect. 3.2).

\subsection{Geodetic influence factors}

The idea is to use an over-determined affine coordinate transformation to assess the movement of the observed points between two subsequent epochs of measurements. This means that the coordinates $\boldsymbol{x}=(x, y)^{T}$ of the points of epoch $\mathrm{n}$ are mapped onto the coordinates $\boldsymbol{x}^{\prime}=\left(x^{\prime}, y^{\prime}\right)^{T}$ of the same points of epoch $n+1$ (for details see e.g. Becker and Bürger, 1975).

$\boldsymbol{x}^{\prime}=\boldsymbol{F} \cdot \boldsymbol{x}+\boldsymbol{t}$

$$
\begin{aligned}
& \text { where } \boldsymbol{x}=(x, y)^{T} \quad \ldots \text { coordinates of epoch } \mathrm{n} \\
& \boldsymbol{x}^{\prime}=\left(x^{\prime}, y^{\prime}\right)^{T} \quad \ldots \text { coordinates of epoch } n+1 \\
& \text { (after deformation) } \\
& \boldsymbol{t}=\left(t_{x}, t_{y}\right)^{T} \quad \ldots \text { translational parameters } \\
& \boldsymbol{F}=\left(\begin{array}{ll}
\frac{\partial x^{\prime}}{\partial x} & \frac{\partial x^{\prime}}{\partial y} \\
\frac{\partial y^{\prime}}{\partial x} & \frac{\partial y^{\prime}}{\partial y}
\end{array}\right) \text {... matrix of deformation }
\end{aligned}
$$

A group of points moving in the same direction (assuming that they are lying on one common block) is characterized by a small range of the residuals between the original and the transformed coordinates and a small standard deviation of unit weight $s_{0}$ within an over-determined affine coordinate transformation. On the contrary, in case points of different blocks were considered simultaneously the standard deviation of unit weight $s_{0}$ and other indicators are significantly larger.

For practical reasons it is better to work with strain parameters instead of the transformation parameters. Since the strain analysis is analogous to the affine coordinate transformation, parameters can easily be converted into the infinitesimal strain components $e_{x x}, e_{y y}$ (rate of change of length per unit length in direction of $\mathrm{x}$-axis respectively $\mathrm{y}$-axis), $e_{x y}$ ( $=e_{y x}$, rate of shear strain) and the derived rotation angle $\omega$ (see e.g. Haberler, 2005).

A direct method of an over-determined computation of these strain parameters can follow this scheme:

$u_{x_{i}}=x_{i}^{\prime}-x_{i}=e_{x x} \cdot x_{i}+e_{x y} \cdot y_{i}+\omega \cdot y_{i}+t_{x}$

$u_{y_{i}}=y_{i}^{\prime}-y_{i}=e_{x y} \cdot x_{i}+e_{y y} \cdot y_{i}-\omega \cdot x_{i}+t_{y}$

resp.

$\boldsymbol{u}=\boldsymbol{H} \cdot \boldsymbol{p}$

where

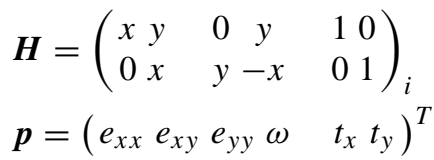

Since the strain parameters are dependent on the coordinate system it is better to transform them into the principal strain axes system, represented by the strain ellipse (Tissot indicatrix). The elements of the strain ellipse (the semi-axes $e_{1}$, $e_{2}$ and the orientation $\theta$ of the maximum strain rate), which fully describe the state of deformation, are calculated from the strain parameters analogous to the geodetic point error ellipse (for further explanations see e.g. Welsch et al., 2000):

$e_{1}=\frac{1}{2}\left(e_{x x}+e_{y y}+e\right)$

$e_{2}=\frac{1}{2}\left(e_{x x}+e_{y y}-e\right)$

$\tan 2 \theta=\frac{2 e_{x y}}{e_{x x}-e_{y y}}$

with $e^{2}=\left(e_{x x}-e_{y y}\right)^{2}+4 e_{x y}^{2}$.

These indicators used as input parameters in the fuzzy system can be determined by the results of the sequence of affine transformations, using a minimum of four points (cf. Sect. 3.3). The following basic example (Fig. 6) shows the different values of the strain ellipse parameters $e_{1}, e_{2}$, and the standard deviation of unit weight $s_{0}$ in two cases: in the left figure four points with almost identical displacement vectors are analysed, in the figure to the right the results for three points with equal and one point with a very different displacement vector are shown. Note the different scales of the strain ellipse parameters. It shows that these parameters can be used as indicators for a block separation.

\subsection{Visual influence factors}

Humans, not only experts, are able to determine blocks of similar pattern of movement, simply by looking at the graph 

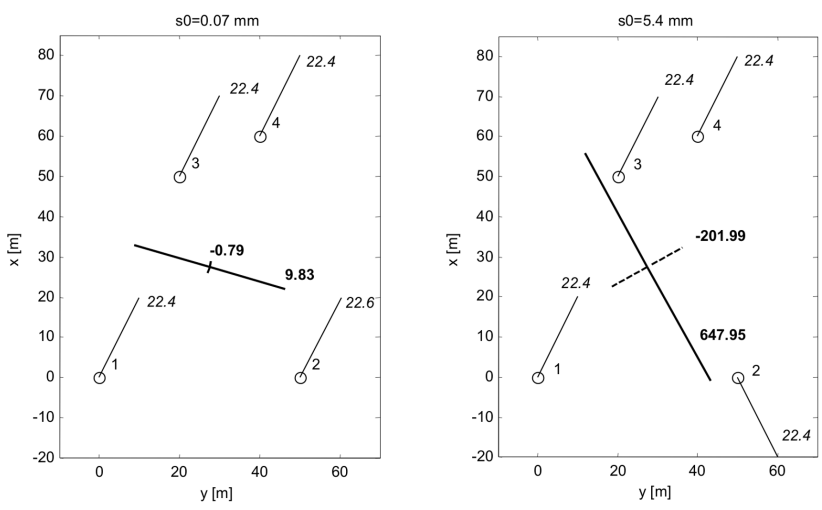

Fig. 6. Indicators $\left(s_{0}\right.$ and $\left.e_{1}, e_{2}\right)$ for two different cases. Displacements (italic, in mm), strain ellipse parameters $e_{1}$ and $e_{2}$ (bold, in ppm).

of displacement vectors. The similarity of the length and the direction of the vectors is assessed on the one hand, and the property of neighbourhood on the other hand. This ability is imitated in the block detection algorithm.

The direction of the displacement vectors is used as a first important input variable. According to human thinking it seems to be clear that only vectors showing similar directions can belong to one common block. The length of the displacement vectors is used as another intuitive input parameter. Since the analysis is done in 2-D, the inclination of the slope has to be taken into account for this parameter. Like before, two or more vectors are said to be similar if the lengths of the displacement vectors are similar. The combination of the direction and the length of the vectors gives a clear distinction which points show the same pattern of movement. In addition, the property of "neighbourhood" is determined from a Delaunay triangulation.

The example shows the modelling of the variable "similarity of direction" (Fig. 5). If the directions of several vectors under investigation are within a range of approximately 20 gon, they are assessed as "similar" by the fuzzy system. The greater the difference in azimuth gets, the smaller the property of "similarity".

\subsection{General algorithm}

The block detection algorithm starts with finding all possible blocks consisting of four neighbouring points (see Fig. 7). A minimum of four points per block is necessary due to the over-determined calculation of the strain analysis. For all these combinations of 4 neighbouring points, the affine transformations are calculated and assessed by the first fuzzy system using the input parameters shown above (strain ellipse parameters, length and direction of the displacement vectors etc.). The first fuzzy system chooses the "best" set according to the input parameters and in an iterative process the best fitting neighbouring points are determined by a sequence of affine transformations (using 5, 6, 7, .. points in these transformations). This iterative process is done until one of the

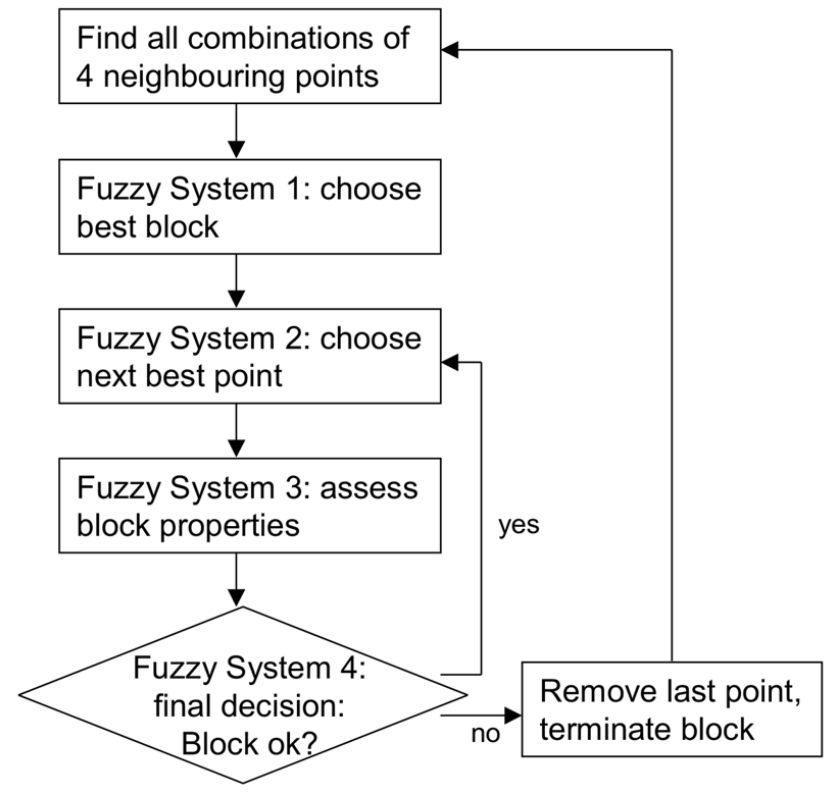

Fig. 7. General scheme of the block detection algorithm.

following fuzzy systems determines that the block is complete, i.e. that no neighbouring points with a similar pattern of movement exist. Then the algorithm starts again finding four neighbouring points out of the remaining points.

The algorithm uses four different fuzzy systems, depending on the task and on the available parameters (Fig. 7):

1. In the first step of the algorithm a multitude of 4-pointcombinations is formed. The first fuzzy system assesses the block quality of the various possibilities using the parameters standard deviation of unit weight $s_{0}$, the axes of the strain ellipse $e_{1}$ and $e_{2}$, the direction and length of the four displacement vectors; the optimal combination is chosen as the starting block.

2. Based on this starting block, in an iterative process the next "best" point is chosen to join the block. The algorithm tries to find all neighbouring points to the starting block. From all possibilities the second fuzzy system has to choose the optimal one by again assessing the parameters $s_{0}, e_{1}, e_{2}$, direction and length of the displacement vectors.

3. The second fuzzy system only chooses the optimal combination out of the several possibilities, as mentioned before. The third fuzzy system now determines in each step of iteration, if the "optimal" solution found in step 2 is still a valid block. This is done by assessing not only the parameters mentioned above, but further adding parameters like the rates of change of the parameters $s_{0}$, $e_{1}, e_{2}$ between subsequent steps of iteration. The idea behind it is that if a point, which does not belong to the block, is added to the block, the parameters $s_{0}, e_{1}, e_{2}$ will increase so that the rates of change between subsequent steps of iteration will be good indicators. 
Table 1. Sequence of outputs of the third fuzzy system in the several steps of iteration, serving as the basis for the decision of fuzzy system 4 .

\begin{tabular}{llllllll}
\hline & Step 1 & Step 2 & Step 3 & Step 4 & Step 5 & Step 6 & Step 7 \\
\hline Point added & 9 & 8 & 7 & 19 & 16 & 17 & $\mathbf{1 4}$ \\
\hline Output of Fuzzy system 3 & 0.55 & 0.49 & 0.45 & 0.52 & 0.50 & 0.54 & $\mathbf{0 . 7 4}$ \\
\hline
\end{tabular}

4. The last fuzzy system gives the last decision if the block has to be terminated. A clear yes/no-decision must be made. Here again not only the output value of the third fuzzy system, but also the change of these output values are used for the calculation of the final decision value.

\section{Example}

The example given here is based on an existing monitoring network near Varna, Bulgaria (see Pelzer and Milev, 1984). Since the monitoring network was showing no significant movement, the original displacement vectors were slightly modified for the demonstration of the algorithm. A graphical representation of the displacement vectors used is given in Fig. 8.

Looking at this Fig. 8, two blocks can intuitively be distinguished. The first (northern) one consists of stable points on a plateau with small displacements (up to $3 \mathrm{~mm}$ between two measurements). The other points are lying on the unstable part of the area, with movements of approximately $1 \mathrm{~cm}$ between two epochs of measurement.

Starting the algorithm, the best-fitting 4-pointcombination is presented, as calculated from the strain analysis: $2-10-13-15$, with an output value of 0.84 of the first fuzzy system. The output value - within a range of $(0,1)$ - can be interpreted as the "quality" of this block: the higher the output value, the "better" the combination. These four points show a very similar pattern of the direction of the vectors; the small differences in length are causing acceptable small strain parameters.

In the next steps, the following neighbouring points are added iteratively to the block using fuzzy system 2: $9-8-7$ $-19-16-17$.

After the last correct point belonging to block 1 (point number 17), the algorithm determines that out of the remaining neighbouring points, point number 14 would be the best choice. Fuzzy system 3 assesses this situation, finding an indicator of 0.74 that this point should not be added to the block. The output of Fuzzy system 3 can be interpreted as an indicator for the termination of the grouping algorithm: the higher the output value (again within the interval $[0,1]$ ), the higher the necessity to stop the block under investigation. Fuzzy system 4 now confirms this decision by the assessment of the changes of the outputs of system 3 within the iterative processing, since this output, which has in all preceding steps been approx. 0.50 , has suddenly changed to 0.74 (Table 1 ). So the block is terminated.

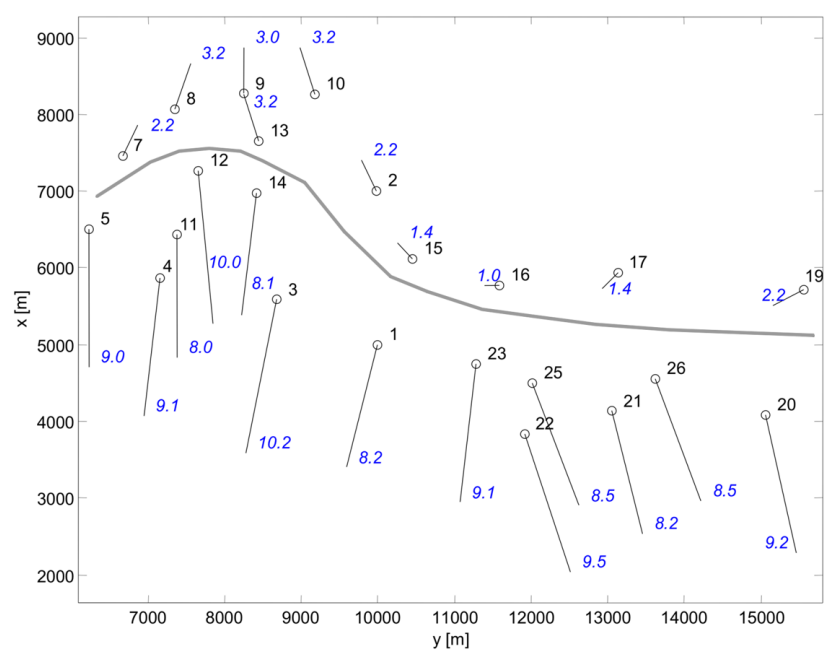

Fig. 8. Graphical representation of the displacement vectors of the example given. The length of the vectors (in $\mathrm{mm}$ ) is given in italic at the end point of the vectors. The grey curve is showing the boundary between the stable and the unstable areas.

Out of the remaining points, the algorithm starts again finding the best 4-point-combination. So, the second block is built up in the following sequence of points: $20-22-25$ $-26-21-4-5-11-12-14-23-1-3$.

Here the algorithm stops because there are no more points available. The algorithm succeeded in extracting the two blocks with different pattern of movement out of the given displacement vectors.

\section{Conclusions}

This algorithm is a further development of the classical geodetic deformation analysis. It is still a pure geometrical analysis, since both, the strain analysis and the visual assessment, are geometrical. No physical parameters of e.g. ground mechanics are considered. An extension of the fuzzy systems taking into account physical and geological parameters is possible.

Acknowledgements. This work was partially supported by the European Commission, Research DG, Environment Programme, Global Change and Natural Disasters.

Edited by: M. Arattano

Reviewed by: T. Wunderlich and another referee 


\section{References}

Becker, E. and Bürger, W.: Kontinuumsmechanik, Teubner Verlag, Stuttgart, 1975.

Haberler, M.: Einsatz von Fuzzy Methoden zur Detektion konsistenter Punktbewegungen, Geowissenschaftliche Mitteilungen, Schriftenreihe der Studienrichtung Vermessungswesen und Geoinformation der Technischen Universität Wien, Heft Nr. 73, 2005.

Heine, K.: Beschreibung von Deformationsprozessen durch Volterra- und Fuzzy-Modelle sowie Neuronale Netze, Deutsche Geodätische Kommission, Reihe C, Heft Nr. 516, Verlag der Bayerischen Akademie der Wissenschaften, München, 1999.

Kruse, R., Gebhardt, J., and Klawonn, F.: Fuzzy Systeme, Teubner Verlag, Stuttgart, 1995.

Lehmkühler, H.: Die geodätische Deformationsanalyse als Mustererkennungsaufgabe, Veröffentlichung des Geodätischen Instituts der Rheinisch-Westfälischen Technischen Hochschule Aachen, Nr. 49, 1993.
Miima, J.-B.: Artificial Neural Networks and Fuzzy Logic Techniques for the Reconstruction of Structural Deformations, Geodätische Schriftenreihe der Technischen Universität Braunschweig, Nr. 18, 2002.

Pelzer, H. and Milev, G. (Eds.): Untersuchungen zur geodätischen Bestimmung von Rutschungserscheinungen und vertikalen Krustenbewegungen, Wissenschaftliche Arbeiten der Fachrichtung Vermessungswesen der Universität Hannover, Nr. 133, 1984.

Pelzer, H. (Ed.): Geodätische Netze in Landes- und Ingenieurvermessung II, Wittwer Verlag, Stuttgart, 1985.

Welsch, W. (Ed.): Deformationsanalysen '83, Schriftenreihe Wissenschaftlicher Studiengang Vermessungswesen Hochschule der Bundeswehr München, Heft Nr. 9, 1983.

Welsch, W., Heuneke, O. and Kuhlmann, H.: Auswertung geodätischer Überwachungs-messungen, Wichmann Verlag, Heidelberg, 2000.

Wieser, A.: Robust and fuzzy techniques for parameter estimation and quality assessment in GPS, Shaker Verlag, Aachen, 2002. 